ARVC or sudden death $(\mathrm{OR}=7.300,95 \%$ CI 1.606 to 33.177, $\mathrm{p}=0.010)$, the accordion sign ( $O R=7.000,95 \%$ CI 1.509 to 32.468 , $\mathrm{p}=0.013$ ) and number of regions with myocardial fibrosis $(\mathrm{OR}=2.204,95 \%$ CI 1.116 to 4.354, $\mathrm{p}=0.023)$ were independent predictors for life-threatening ventricular arrhythmia in ARVC.

Conclusions MRI is the optimal imaging approach for detecting ARVC. Familial history of ARVC or sudden death, the accordion sign and number of regions with myocardial fibrosis were associated with an increased risk of life-threatening ventricular arrhythmia in patients with ARVC.

\section{e0605 "ONE-STOP SHOP” EXAMINATION OF COMPLICATED AND COMPLEX CONGENITAL HEART DISEASE WITH MRI}

doi:10.1136/hrt.2010.208967.605

${ }^{1}$ Lu Minjie, 'Zhao Shihua, ' Jiang Shiliang, 'Lv Jianhua, 'Zhang Yan, ${ }^{2}$ Renate Jerrecic. ${ }^{1}$ Fuwai Hospital; '2 2 iemens Healthcare Group

Objective To evaluate the diagnostic value of MRI in complicated congenital heart disease, comparing with echocardiography and $\mathrm{x}$-ray cardio-angiography.

Methods 20 patients with complicated or complex congenital heart disease (male 12, female 8 , mean age $17.8 \mathrm{y}$, ranging from $3-46 \mathrm{y}$ ) underwent MR examination. A wireless vector ECG-gating was used for triggering; all the sequences were applied with breath hold or free breathing. Turbo spin echo and multiple gradient echo sequences including FLASH (fast low angle shot) and TrueFISP (true fast imaging with steady-state precession.) with TSENSE (adaptive sensitivity encoding incorporating temporal filtering) and TREAT (time resolved echo-shared technique) were used to evaluate the morphology, function, flow status of the heart. MR findings were compared with the results of echocardiography and x-ray cardio-angiography.

Results All the examination were accomplished successfully and diagnostic $M R$ images were obtained satisfactory. The mean total scan time was $38 \mathrm{~min}$ ranging from $29 \sim 55 \mathrm{~min}$. According to the main malformation diagnosed by MRI, double-outlet of right ventricle (DORV) were 11 cases, single ventricle were 3 cases, function-corrected transposition of great arteries were 2 cases, endocardial cushion defect, interruption of aortic arch, coarctation of the aorta and pulmonary atresia was 1 case, respectively. The total coincidence was $95 \%$ comparing with cardio-angiography, while the echocardiography was $75 \%$. It is difficult for $\mathrm{x}$-ray cardioangiography to evaluate the atrio-rentriculor valve in $25 \%$ cases, while MR can clearly and precisely show the number, morphology and function of the valves in all cases. There is $20 \%$ of the cases that traditional $\mathrm{x}$-ray cardio-angiography cannot demonstrate the connection of ventricular-great arteries, or the main pulmonary artery and its main branches because of anatomy overlapping, tolerance of the patients and skill of catheterisation, while MR provide important complementarities for these cases.

Conclusion Combined with new robust techniques, MRI can provide a comprehensive evaluation of complicated congenital heart disease including morphology, function, and flow and so on. With some characteristics of both echocardiography and x-ray angiography, in some aspects MRI is even better than x-ray angiography and can offer important supplemental information.

\section{e0606 TRANSCATHETER CLOSURE OF A GIGANTIC RIGHT SINUS OF VALSALVA ANEURYSM TO RIGHT VENTRICULAR FISTULA: A CASE REPORT}

doi:10.1136/hrt.2010.208967.606

Wang Haiyan, Zheng Qinagsun, Xue Yusheng, Liu Yin. Department of Cardiology, Tangdu Hospital, The Fourth Military Medical University

Ruptured aneurysms of the sinus of Valsalva with a gigantic fistulous tract are extremely rare. A single origin of the coronary arteries is a rare coronary anomaly. We describe the case of a 6-year-old girl who had a ruptured aneurysm of the right coronary sinus with a gigantic fistulous tract and an anomalous origin of the right coronary artery. Transthoracic echocardiography showed the right coronary artery (RCA) aneurysm and RCA fistula connecting to the right ventricular (RV). Aortography revealed the presence of a right coronary sinus of Valsalva aneurysm (SVA)-RV fistula and the originating of right coronary artery (RCA) from left circumflex artery (LCX). She was diagnosed as having a gigantic SVA to right ventricle fistula and a single origin of the coronary arteries. Successful trancatheter closure of the fistulous ostium was performed.

\section{E0607 ANALYSIS ON EARLY DIAGNOSIS GRADING MODEL OF ACUTE AORTIC DISSECTION}

doi:10.1136/hrt.2010.208967.607

Liu Qiming, Tang Ming, Zhou Shenghua, Chai Xiangping. Department of emergency, Second Xiangya Hospital, Central South University, Changsha, Hunan

Objective To investigate the features of the clinical manifestation, laboratory data and imageology information in acute Aortic Dissection $(\mathrm{AD})$ patients, to find the early, right and easy grading model of diagnosising $\mathrm{AD}$.

Method Analyze the clinical manifestation, Laboratory data and imageology information of $182 \mathrm{AD}$ patients, who were our patients at Emergency Department in last three years, and meanwhile, compared them with 184 chest and back ache patients, to search the early diagnosis grading model of $\mathrm{AD}$

Results If 5 score was the standard of the grading system to early diagnose $\mathrm{AD}$ on the basis of logistic regression equation and clincal practice, the sensitivity of $\mathrm{AD}$ forecast is $96.7 \%$, specificity is $81.0 \%$. Conclusions We can improve the emergency diagnose level of $\mathrm{AD}$ through establishing the early grading model which contains the stabbing and severe pain, distinct rise of the blood pressure, asymmetry of the blood pressure and/or the pulse, wide arteriae aorta and/or mediastinum on chest x-ray, obvious rise of D-dimmer.

\section{E0608 A NITINOL OCCLUDER AND A SPECIAL DELIVERY DEVICE FOR PATENT DUCTUS ARTERIOSUS (PDA) CLOSURE}

doi:10.1136/hrt.2010.208967.608

Jiang Hai-bin, Zhao Xian-xian, Zong Gang-jun, Bai Yuan, Han Lin, Qin Yong-wen Changhai Hospital

Background Nitrol occluders used for patent ductus arteriosus (PDA) may result in the stenosis of aortic and pulmonary artery when applied for smaller-sized children. The present study sought to design and develop an improved nitinol occluder and delivery system, and to verify the reliability and safety of this device on canine model of PDA

Methods A canine PDA model was established by anastomosing the internal jugular vein with the left pulmonary artery and the descending aorta in an end-to-side fashion. The effects of the novel nitinol occluder and its delivery system were followed-up for 6 months after the closure.

Results PDA was successfully established in 10 out of 18 dogs and was treated by transcatheter occlusion with the novel nitinol occluder. Postoperative echocardiography showed that the location and shape of the occluder were normal without any regurgitation. Morphological examination revealed that the surface of the occluder was covered by a grey membrane-like tissue, which was identified as endothelial tissue by histological and electron microscopy examinations. There was no corrosion or severe inflammation on the 